\title{
Inhibition of STAT3 acetylation is associated with attenuated renal fibrosis in the obstructed kidney
}

\author{
Jun $\mathrm{NI}^{1}$, Yang SHEN ${ }^{1}$, Zhen WANG ${ }^{1}$, De-cui SHAO ${ }^{1}$, Jia LIU ${ }^{1}$, Lan-jun FU ${ }^{2}$, Ya-li KONG ${ }^{1}$, Li ZHOU ${ }^{1}$, Hong XUE ${ }^{1}$, Yu HUANG ${ }^{3}$, \\ Wei ZHANG ${ }^{1}$, Chen $\mathrm{YU}^{2, *}$, Li-min $\mathrm{LU}^{1, *}$ \\ ${ }^{1}$ Department of Physiology and Pathophysiology, Shanghai Medical College, Fudan University, Shanghai 200032, China; ${ }^{2}$ Department \\ of Nephrology, Tongji Hospital, Tongji University School of Medicine, Shanghai 200065, China; ${ }^{3}$ School of Biomedical Sciences and \\ Institute of Vascular Medicine, Chinese University of Hong Kong, Hong Kong, China
}

\begin{abstract}
Aim: To explore the relationship between the signal transducer and activator of transcription 3 (STAT3) signaling and renal fibrosis. Methods: Rat renal tubular epithelial NRK-52E cells were treated with angiotensin II (Ang II), nicotinamide (an inhibitor of NAD'dependent class III protein deacetylases, SIRT1-7), or resveratrol (an activator of SIRT1). Mice underwent unilateral ureteral obstruction (UUO) were used for in vivo studies. Renal interstitial fibrosis was observed with HE and Masson's trichrome staining. STAT3 acetylation and phosphorylation, fibronectin, collagen I, collagen IV, and $\alpha$-smooth muscle actin ( $\alpha$-SMA) levels were examined using Western blotting.

Results: Nicotinamide $(0.625-10 \mathrm{mmol} / \mathrm{L})$ dose-dependently increased STAT3 acetylation on Lys 685 and phosphorylation on Tyr705 in NRK-52E cells, accompanied by accumulation of fibronectin and collagen IV. Ang II increased STAT3 phosphorylation on Tyr705 and the expression of fibronectin, collagen IV and $\alpha$-SMA in the cells. Pretreatment with resveratrol $(12.5 \mu \mathrm{mol} / \mathrm{L})$ blocked Ang II-induced effects in the cells. UUO induced marked STAT3 phosphorylation, fibronectin, collagen IV and $\alpha$-SMA accumulation, and renal interstitial fibrosis in the obstructed kidneys, which were significantly attenuated by daily administration of resveratrol (100 mg/kg). Conclusion: STAT3 acetylation plays an important role in activation of STAT3 signaling pathway and consequent renal fibrosis.
\end{abstract}

Keywords: chronic kidney disease; renal fibrosis; unilateral ureteral obstruction (UUO); angiotensin II; resveratrol; nicotinamide; STAT3; acetylation; phosphorylation

Acta Pharmacologica Sinica (2014) 35: 1045-1054; doi: 10.1038/aps.2014.42; published online 30 Jun 2014

\section{Introduction}

Renal fibrosis, which is characterized by glomerulosclerosis and tubulointerstitial fibrosis, has been considered as a typical pathological feature during the progression of chronic kidney disease $(\mathrm{CKD})^{[1,2]}$. Although there are various apparent causes of renal fibrosis, this pathological feature may induce irreversible dysfunction of the kidney and eventually leads to end-stage kidney disease. Despite the debate whether injured epithelial cells can be the direct precursor of myofibroblasts via processing type II epithelial-to-mesenchymal transition (EMT), renal tubular epithelial cell is known as a primary cell in which pro-fibrotic cellular changes occur, contributing to

\footnotetext{
* To whom correspondence should be addressed.

E-mail yuchen2001@hotmail.com (Yu CHEN) lulimin@shmu.edu.cn (Li-min LU)

Received 2013-12-03 Accepted 2014-04-25
}

the early development and progression of renal interstitial fibrosis $^{[3-9]}$. These changes include decrease in tubular diameter, increase in expression of a-smooth muscle actin (a-SMA) and deposition of extracellular matrix, ultimately leading to tubular atrophy, loss of functional renal parenchyma, which contribute to renal interstitial fibrosis.

Although the exact mechanism of renal interstitial fibrosis is unclear, increasing evidence suggests that the excessive activation of the local renin-angiotensin system (RAS), which leads to a prominent elevation of angiotensin II (Ang II), is involved in the progression of $\mathrm{CKD}^{[10-12]}$. Previous in vivo studies have shown that the elevation of Ang II by chronic infusion results in renal fibrosis, which is associated with expression of proinflammatory cytokines and fibrosis-associated genes ${ }^{[13]}$. Ang II is one of the main pathogenic mediators of renal interstitial fibrosis, especially in obstructive nephropathy ${ }^{[10-12,14]}$. Additionally, a number of previous studies have revealed that 
the suppression of intrarenal RAS prevents the pro-fibrotic changes induced by unilateral ureteral obstruction (UUO) ${ }^{[15-17]}$.

The Janus kinase family (JAK)/signal transducers and activators of transcription (STAT) signaling pathway constitutes an important cascade for a wide range of signal transduction cytokines and growth factors expression. The JAK2/STAT3 signaling pathway can be activated by Ang II in different organs, including the kidney ${ }^{[18-22]}$. STAT3 has previously been considered to be involved in promoting cell cycle progression and cellular transformation and in preventing apoptosis ${ }^{[23,24]}$. Increasing evidence suggests that STAT3 can be activated and highly expressed during the progression of fibrogenesis in the obstructed kidney ${ }^{[25-27]}$ and under other pathophysiological circumstances $^{[9]}$. Despite being one of the major mediators of transforming growth factor- $\beta 1$ (TGF- $\beta 1$ )-driven kidney dysfunction ${ }^{[27-29]}$, the role of Ang II-induced STAT3 signaling in renal fibrogenesis remains uncertain.

Although the classical view of STAT3 activation focuses on the phosphorylation on tyrosine 705 (Tyr705) and serine 727 (ser727) ${ }^{[30]}$, recent studies have shown that acetylation on lysine 685 (Lys685) of STAT3 also plays a role in transactivation of target genes ${ }^{[31,32]}$. The reversible acetylation of STAT3 is regulated by the CREB-binding protein/p300 family of histone acetyltransferases and histone deacetylases (HDACs) ${ }^{[31,33]}$. Several HDACs have been reported to be involved in deacetylation of STAT3 ${ }^{[2,34]}$. One of the best characterized deacetylases is sirtuin 1 (SIRT1), a member of nicotinamide adenine dinucleotide $\left(\mathrm{NAD}^{+}\right)$-dependent class III protein deacetylases, which can be inhibited by nicotinamide ${ }^{[35,36]}$. SIRT1, which mediates the deacetylation of STAT3 Lys685 site ${ }^{[37-41]}$, can be activated by resveratrol (trans-3,4',5-trihydroxystilbene) ${ }^{[42,43]}$, a natural polyphenol with renal protective effects that depend on deacetylation of Smad3 $3^{[44]}, \mathrm{p} 53^{[45]}$, and NF- $\mathrm{KB}$ p65 $5^{[46]}$. However, the role of STAT3 acetylation in the antifibrotic activity of resveratrol has yet to be clarified.

In the present study, we investigated the relationship between the acetylation and phosphorylation of STAT3 in Ang II-induced pro-fibrotic responses in renal tubular epithelial cells and UUO-induced renal fibrosis to determine the role of STAT3 signaling in renal fibrogenesis both in vitro and in vivo.

\section{Materials and methods Materials}

Dulbecco's modified Eagle's medium (DMEM), human angiotensin II, nicotinamide and resveratrol were purchased from Sigma-Aldrich (Saint Louis, MO, USA). Fetal bovine serum (FBS) was obtained from Tianhang Biotechnology Co (Hangzhou, China). The BCA Protein Assay Kit was from Shenergy Biocolor BioScience and Technology (Shanghai, China). Antifibronectin antibody was obtained from Sigma-Aldrich (Saint Louis, MO, USA), and anti-a-SMA antibody, from Santa Cruz Biotechnologies, Inc (Santa Cruz, CA, USA). Anti-collagen I antibody and anti-collagen IV antibody were procured from Abcam (Cambridge, MA, USA). Anti-phospho-STAT3 (Tyr705) antibody, anti-phospho-STAT3 (Ser727) antibody,
anti-acetyl-STAT3 (K685) antibody and anti-STAT3 antibody were obtained from Cell Signaling Technology (Beverly, MA, USA). Anti-GAPDH antibody, anti- $\beta$-actin antibody, horseradish peroxidase-conjugated secondary antibodies and enhanced chemiluminescence (ECL) detection kit were obtained from Beyotime Institute of Biotechnology (Haimen, China). Polyvinylidene difluoride membrane was obtained from Millipore (Billerica, MA, USA). Protease and phosphatase inhibitors were from Roche (Mannheim, Germany). All of the other chemicals and reagents used were of analytical grade.

\section{Animals}

Male C57BL/6J mice (20-25 g) were obtained from Shanghai SLAC Laboratory Animal Co Ltd (Shanghai, China). All animal experiments were performed according to the Criteria of the Medical Laboratory Animal Administrative Committee of Shanghai and the Guide for Care and Use of Laboratory Animals of Fudan University, and the protocols were approved by the Ethics Committee for Experimental Research, Shanghai Medical College, Fudan University. UUO surgery was performed under $10 \%$ chloral hydrate anesthesia via intraperitoneal injection $(4 \mathrm{~mL} / \mathrm{kg}$ body weight). The left ureter was visualized by a flank incision and ligated with $5-0$ silk. Sham mice underwent the same surgery, except the left ureter ligation. Over the course of the study, six mice were included in each group and were euthanized 1, 3, or $7 \mathrm{~d}$ after surgery. In the following study, four mice were used in each group. Mice that underwent UUO surgery were treated either with vehicle $(0.5 \%$ carboxymethyl cellulose) or resveratrol $(100 \mathrm{mg} / \mathrm{kg}$ body weight) through oral gavage at $0.1 \mathrm{~mL}$ per day until they were euthanized at the end of the experiment.

\section{Cell culture}

The rat renal tubular epithelial cell line (NRK-52E) was purchased from the Institute of Biochemistry and Cell Biology (Shanghai, China) and cultured in DMEM media containing $10 \% \mathrm{FBS}$ in an atmosphere of $5 \% \mathrm{CO}_{2}$ at $37^{\circ} \mathrm{C}$. Before the experiment, cells were starved in a medium containing $0.5 \%$ FBS for $24 \mathrm{~h}$ to obtain quiescent cells. Cells were treated with Ang II at different concentrations for a designated time period before they were harvested. When necessary, cells were pretreated with diluent (DMSO) or resveratrol $(12.5 \mu \mathrm{mol} / \mathrm{L})$ for $60 \mathrm{~min}$ before Ang II application. Each experiment was repeated at least three times.

\section{Western blotting}

Cell lysate homogenates were prepared as previously described ${ }^{[4]}$. In brief, the renal cortex or cultured cells were lysed in 1×sodium dodecyl sulfate (SDS) and supplemented with proteinase inhibitor and phosphatase inhibitor. Protein concentrations were determined using a BCA Protein Assay Kit according to the manufacturer's instructions. An equal amount ( $40 \mu \mathrm{g}$ per sample) of total protein was loaded and separated by electrophoresis on a 10\% SDS-PAGE gel and then transblotted onto a polyvinylidene difluoride (PVDF) mem- 
brane. After the transfer, the membrane was blocked with 5\% skim milk powder dissolved in Tris-buffered saline with $0.1 \%$ Tween (TBS/Tween) for $1 \mathrm{~h}$ at room temperature with gentle shaking. The membranes were then incubated with primary antibody overnight at $4^{\circ} \mathrm{C}$ [anti-fibronectin antibody, 1:20000; anti-collagen I antibody, 1:1000; anti-collagen IV antibody, 1:1000; anti-a-SMA antibody, 1:5000; anti-phospho-STAT3 (Tyr705) antibody, 1:1000; anti-phospho-STAT3 (Ser727) antibody, 1:1000; anti-acetyl-STAT3 (K685) antibody, 1:500; antiSTAT3 antibody 1:2000; and anti- $\beta$-actin antibody, 1:5000]. On the next day, the membranes were washed 3 times with TBS/ Tween and incubated with horseradish peroxidase-conjugated secondary antibodies for $2 \mathrm{~h}$. After another 3 washes with TBS/Tween, the hybridizing bands were developed using an ECL detection kit according to the manufacturer's instructions. The signals were then imaged by chemiluminescence for 0.1-5 min to visualize signals. Graphs represent densitometric analyses of bands and were normalized to GAPDH or STAT3 signals, with relative protein levels expressed as fold inductions over controls.

\section{Renal histology}

Kidneys were fixed in 10\% neutral buffered formalin and then embedded in paraffin. Sections were collected and prepared accordingly. Slides of paraffinized tissue sections were deparaffinized, rehydrated and washed in distilled water. Sections were stained with hematoxylin-eosin (HE) and Masson's trichrome. Histological changes, such as the degrees of tubular atrophy and interstitial fibrosis, were observed at $400 \times$ optical magnification.

\section{Statistical analysis}

Data are presented as the mean \pm SEM with statistical analysis performed using one-way analysis of variance with post hoc analysis using Tukey's multiple comparison test. The differences between two groups were compared using Student's t-test. $P<0.05$ was considered to indicate a statistically significant difference between mean values.

\section{Results}

Nicotinamide induced STAT3 activation and pro-fibrotic responses in cultured renal tubular epithelial cells

Western blot studies showed that nicotinamide, an inhibitor of SIRTs ${ }^{[35,36]}$, significantly enhanced STAT3 acetylation on Lys685 and the phosphorylation on Tyr705 in NRK-52E cells (48 h) (Figure 1A). These effects were accompanied by an accumulation of fibronectin and collagen IV (Figure 1B).

\section{Ang II induced STAT3 activation and pro-fibrotic responses in} cultured renal tubular epithelial cells

Western blot analysis showed that the Ang II treatment of NRK-52E cells for $48 \mathrm{~h}$ increased fibronectin, collagen IV and a-SMA protein levels in a dose-dependent manner (Figure 2A). A dose of $1 \mu \mathrm{mol} / \mathrm{L}$ Ang II, which can significantly upregulate the hallmarks of fibrogenesis and ECM, increased STAT3 phosphorylation on Tyr705, peaking at 60 min (Figure
2B). Ang II application did not affect STAT3 phosphorylation on ser727 or acetylation on Lys685 (Figure 2B).

Resveratrol prevented Ang Il-induced STAT3 phosphorylation on Try705 and pro-fibrotic responses in tubular epithelial cells via inhibition of STAT3 acetylation on Lys685

Resveratrol, an activator of SIRT1 ${ }^{[42,43]}$, reduced the basal STAT3 acetylation on Lys685 (Figure 3A). Resveratrol pretreatment of tubular epithelial cells prevented Ang II-induced upregulation of STAT3 phosphorylation on Tyr705 (Figure 3A). Neither resveratrol nor Ang II had an obvious effect on STAT3's ser727 phosphorylation (Figure 3A). Furthermore, resveratrol attenuated Ang II-induced upregulation of fibronectin, collagen I, collagen IV, and a-SMA (Figure 3B).

Resveratrol inhibited STAT3 acetylation, phosphorylation and renal fibrosis in the obstructed kidney

In UUO mice, a significant increase in STAT3 phosphorylation on Tyr705 was observed $1 \mathrm{~d}$ after surgery, whereas no obvious change in STAT3 acetylation on Lys685 was found (Figure 4A). The fibrotic associated proteins, including fibronectin, collagen IV and a-SMA were increased in UUO mice and showed statistical significance starting on the third day after surgery (Figure 4B).

Resveratrol treatment of UUO mice through gavage decreased STAT3 acetylation on Lys685 (Figure 5A). This effect was accompanied by a reduction in UUO-induced Tyr705 phosphorylation ( $1 \mathrm{~d}$ ) (Figure 5A). Resveratrol also inhibited UUO-induced upregulation of fibronectin, collagen I, collagen IV, and a-SMA protein levels in the obstructed kidney (3 d) (Figure 5B).

HE staining was performed to observe the histological changes in the kidney. Compared with the vehicle and sham kidneys, the obstructed kidneys showed interstitial fibrosis at d 3. Resveratrol treatment appeared to ameliorate UUOinduced renal fibrosis (Figure 6A). Masson's trichrome staining showed that collagen accumulation (blue stains) was also attenuated by resveratrol treatment (Figure 6B). These results were consistent with the in vitro findings, which suggested that STAT3 acetylation was involved in the pro-fibrotic action of the obstructed kidney.

\section{Discussion}

The major STAT3 acetylation site that enhances its DNA binding activity and transactivation activity is located at its C-terminal position lysine $685^{[31]}$. The present study demonstrated a novel mechanism of fibrogenesis mediated by STAT3 acetylation that had not been previously reported in the kidney. STAT3 acetylation on Lys685 is necessary for STAT3 signaling and the transactivation of downstream pro-fibrotic genes.

The class III deacetylases, silent information regulator 2 (Sir2) family histone/protein deacetylases (sirtuins or SIRTs), catalyze the $\mathrm{NAD}^{+}$-dependent deacetylation of histone and non-histone proteins ${ }^{[35,48-50]}$. In this study, we used nicotinamide, an inhibitor of SIRTs ${ }^{[35,36]}$, to investigate the role of STAT3 acetylation in the progression of tubulointerstitial 
A

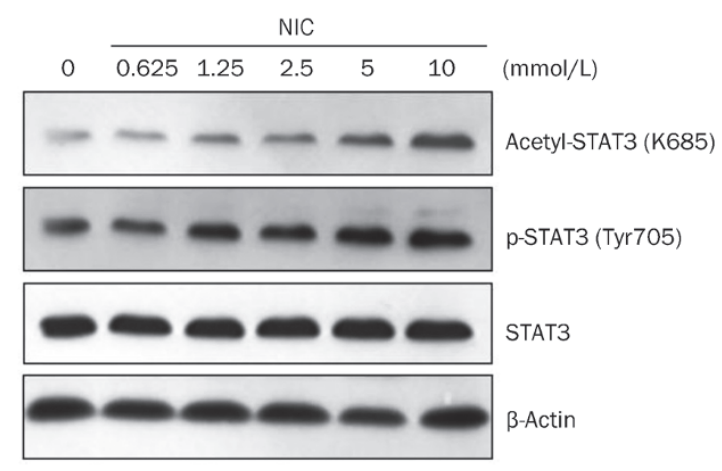

B

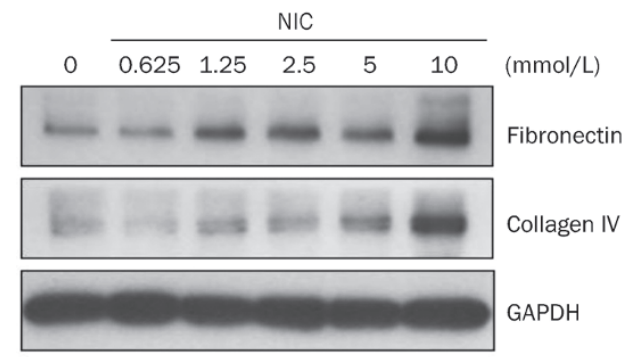

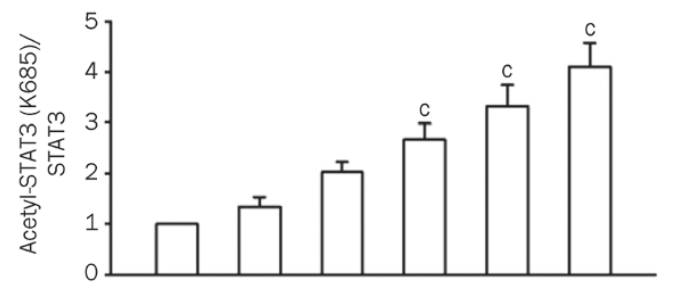

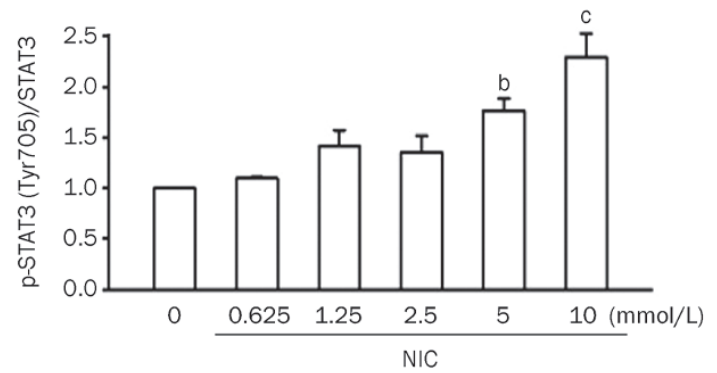

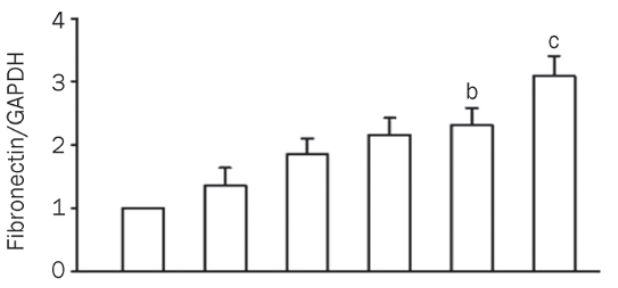

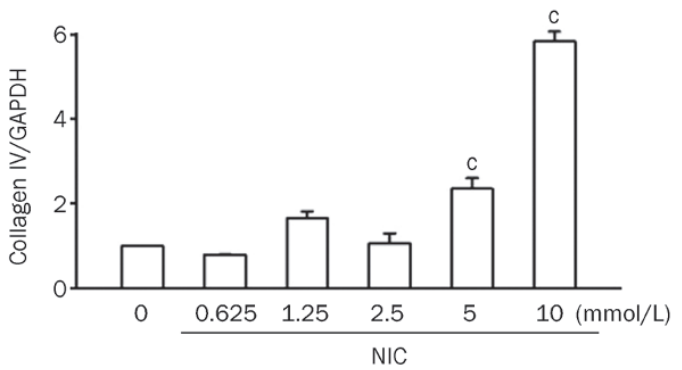

Figure 1. Effect of nicotinamide (NIC) on signal transducer and activator of transcription 3 (STAT3) activation and extracellular matrix (ECM) expression in NRK-52E cells. Cells were incubated with NIC at the indicated dose for $48 \mathrm{~h}$. (A) Western blotting visualized the levels of acetyl-STAT3 (Lys685) and phospho-STAT3 (Tyr705). (B) Fibronectin and collagen IV levels were detected by Western blotting. Media containing NIC were changed every $24 \mathrm{~h}$. Data are the mean \pm SEM of $3-6$ experiments. ${ }^{\mathrm{b}} P<0.05,{ }^{\mathrm{C}} \mathrm{P}<0.01$ compared with control [NIC $\left.(0 \mathrm{mmol} / \mathrm{L})\right]$.

fibrosis. The present in vitro study showed that nicotinamide increased both STAT3 acetylation and phosphorylation, concomitant with the enhanced pro-fibrotic gene expression in tubular epithelial cells. These changes that had not been reported previously indicated the correlation between STAT3 signaling and renal fibrosis. Therefore, the most critical question remains as to the role of STAT3 acetylation and phosphorylation in pro-fibrotic responses.

To answer this question, we investigated the role of STAT3 activation in cellular pro-fibrotic responses by Ang II stimulation in cultured proximal tubular epithelial cells. Ang II has been demonstrated to be involved in multiple pathogenesisinduced renal fibrosis, while STAT3 signaling has been observed to be involved in Ang II-induced renal tubular epithelial cell dysfunction. For example, STAT3 is involved in the Ang II-induced expression of tissue inhibitor of metalloproteinase-1 (TIMP-1) in proximal tubular epithelial cells ${ }^{[22]}$. However, the exact role of STAT3 in Ang II-induced renal pro-fibrotic changes has not been thoroughly investigated. We observed that Ang II induced pro-fibrotic responses in a dose-dependent fashion, which was consistent with previ- ous observations. Since nicotinamide can increase the basal level of STAT3 acetylation through the inhibition of SIRT1, we treated cells with nicotinamide for $48 \mathrm{~h}$ and detected a simultaneous increase of the phosphorylation on Tyr705. The longlasting progress of nicotinamide was different from the effect of Ang II, which can upregulate STAT3 phosphorylation on Tyr705 by direct activation of JAK2, the upstream tyrosine kinase of STAT3. Thus, we detected Ang II/STAT3 signaling in a classical short time-dependent fashion within $2 \mathrm{~h}$. Ang II treatment increased STAT3 Try705 phosphorylation, suggesting that STAT3 signaling might be involved in Ang II-induced functional changes in tubular epithelial cells. However, no obvious change in STAT3 Ser727 phosphorylation or Lys685 acetylation was shown. These findings indicated that the effect of Ang II on STAT3 signaling was selective.

SIRT1 has been proven to be the major enzyme that removes acetyl groups from STAT3 lysine residues ${ }^{[38,39,41]}$. As a SIRT1 activator ${ }^{[42,43]}$, resveratrol was used to identify whether acetylated STAT3 is involved in the activation of STAT3 signaling. The basal STAT3 acetylation on Lys685 was decreased by resveratrol, resulting in negation of Ang II-induced upregulation 
A

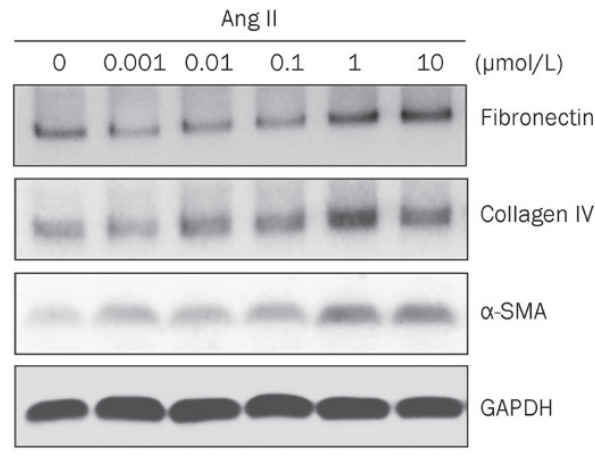

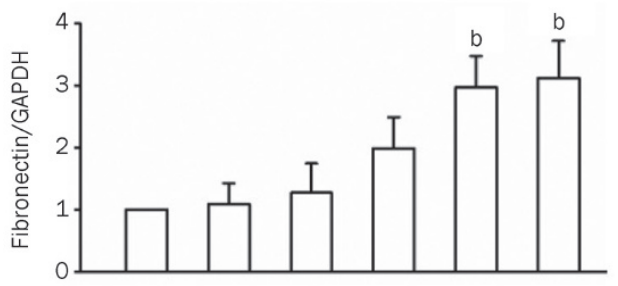
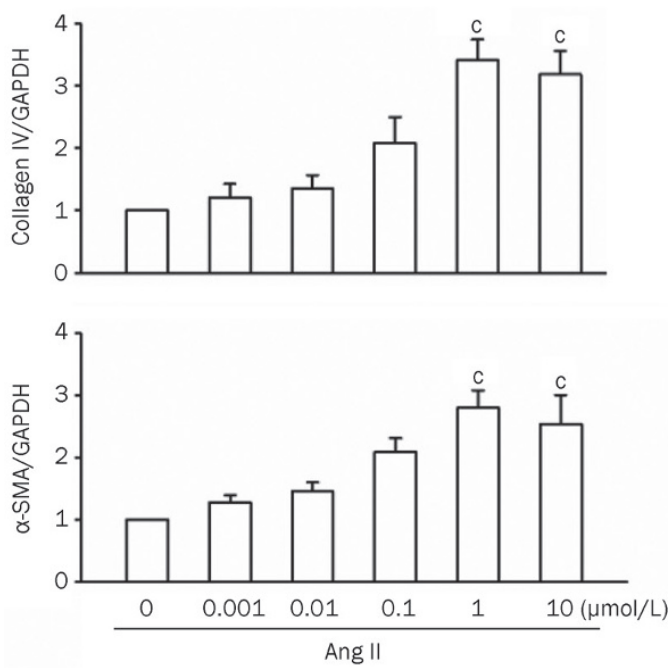

B
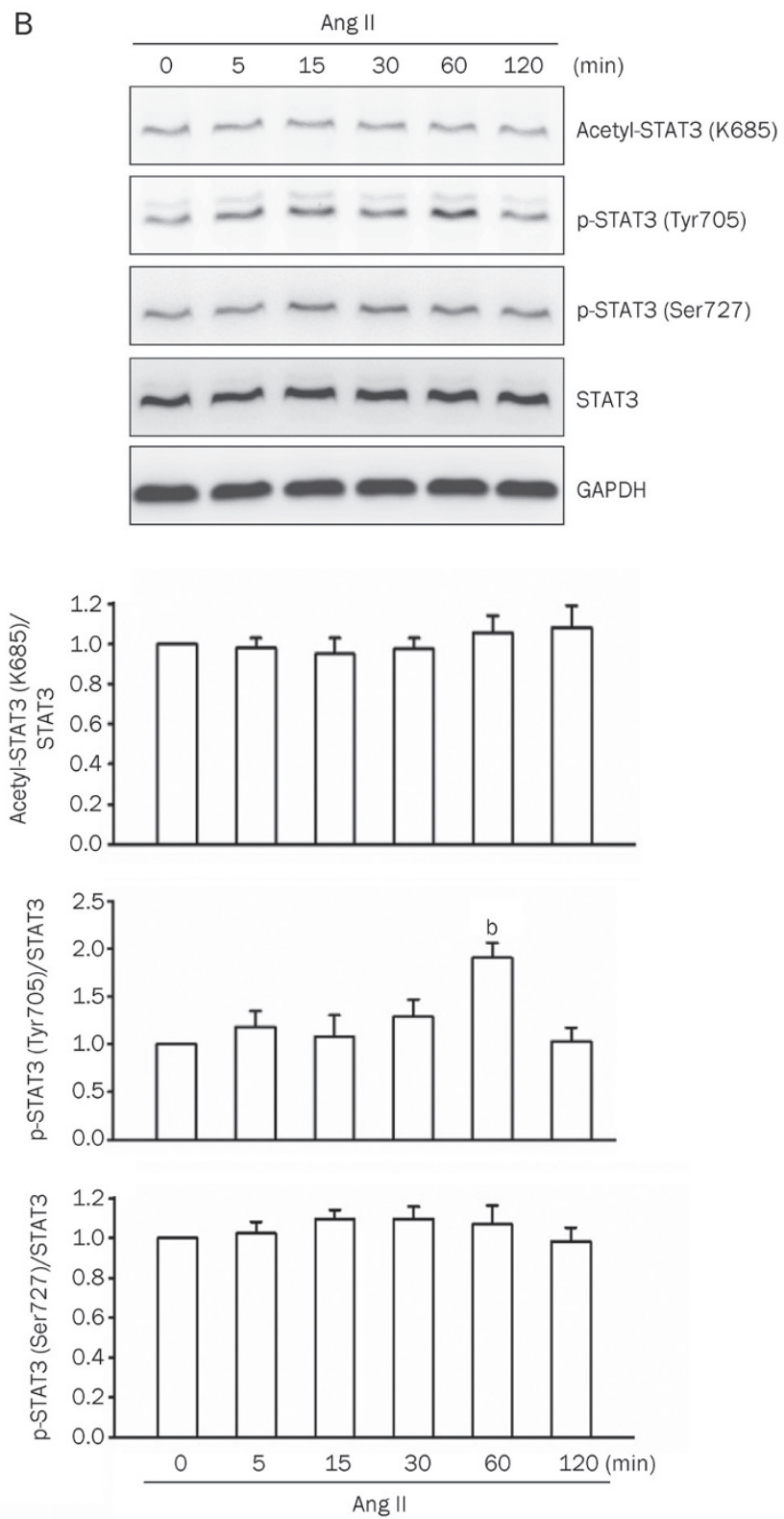

Figure 2. Effect of angiotensin II (Ang II) on STAT3 activation and expression of pro-fibrotic genes in NRK-52E cells. (A) Cells were treated with Ang II at the indicated concentrations for $48 \mathrm{~h}$. Fibronectin, collagen IV and $\alpha$-smooth muscle actin $(\alpha-S M A)$ were analyzed by Western blot analysis. Media containing Ang II were changed every $24 \mathrm{~h}$. (B) Cells were stimulated with $1 \mu \mathrm{mol} / \mathrm{L}$ Ang II for 0-120 min. Acetyl-STAT3 (Lys685), phospho-STAT3 (Tyr705) and phospho-STAT3 (Ser727) were analyzed by Western blot analysis. Data are the mean \pm SEM of $4-5$ experiments. ${ }^{b} P<0.05,{ }^{\mathrm{c}} P<0.01$ compared with control [Ang II ( $0 \mu \mathrm{mol} / \mathrm{L})$ or Ang II (0 min)].

of STAT3 Try705 phosphorylation and the downstream profibrotic genes. Furthermore, resveratrol did not affect STAT3 phosphorylation on Ser727, indicating that the action of this treatment was selective specifically in the STAT3 signaling pathway. Thus, we speculate that STAT3 acetylation on Lys685 is necessary for activation of STAT3 signaling involved in Ang II-induced pro-fibrotic changes of tubular epithelial cells. However, the relationship between acetylation on Lys685 and phosphorylation on Try705 still requires further investigation.

In obstructed kidneys, Ang II levels have been proven to be markedly elevated ${ }^{[10,11]}$, whereas Ang II type 1 receptor blocker (ARB) and angiotensin-converting enzyme inhibitor (ACEI) demonstrate significant antifibrotic effects ${ }^{[15,17]}$. Furthermore, the formation of renal cortical TGF- $\beta 1$ and related fibrogenic factors can be considerably reduced by angiotensinogen antisense RNA treatment in early $\mathrm{UUO}^{[16]}$. Thus, the effect of STAT3 acetylation inhibition on antifibrotic actions was further determined in UUO mice. UUO significantly increased STAT3 phosphorylation on Try705 in the early stage of renal fibrosis, and this modification was partially inhibited by resveratrol. Moreover, resveratrol treatment exhibited an antifi- 
A

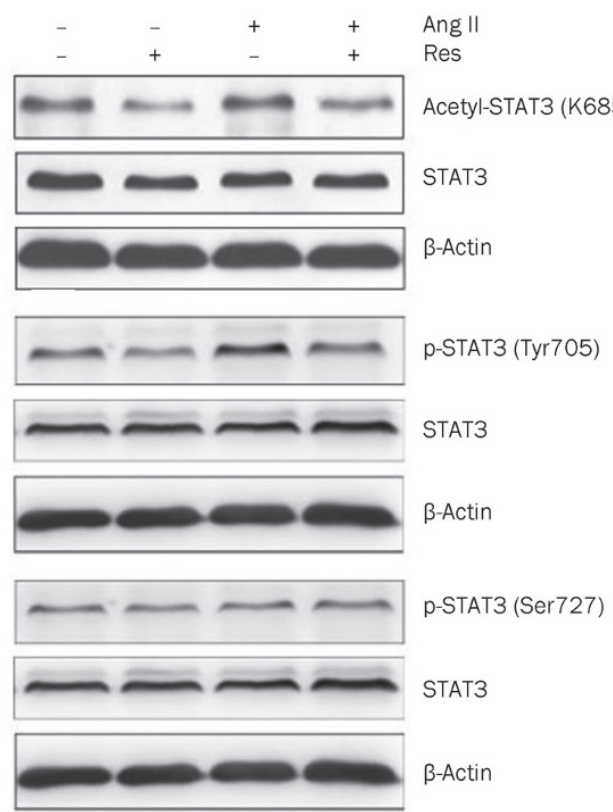

B
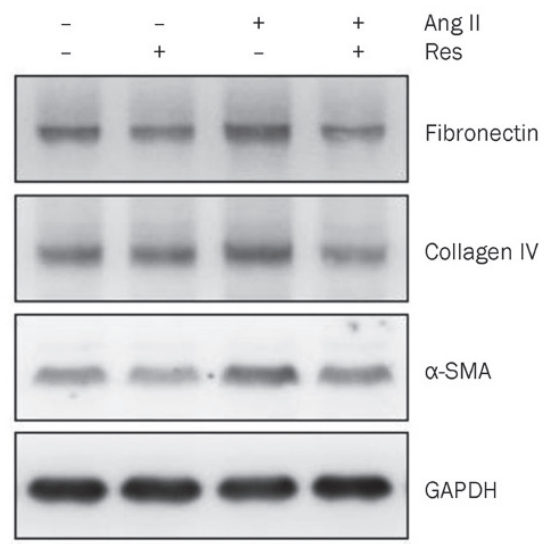

$\alpha-S M A$

GAPDH

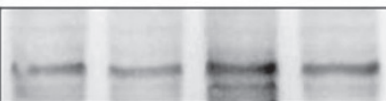

Collagen I

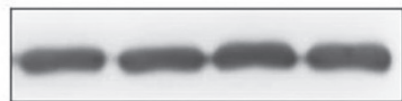

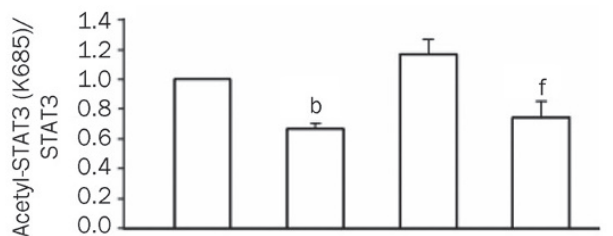
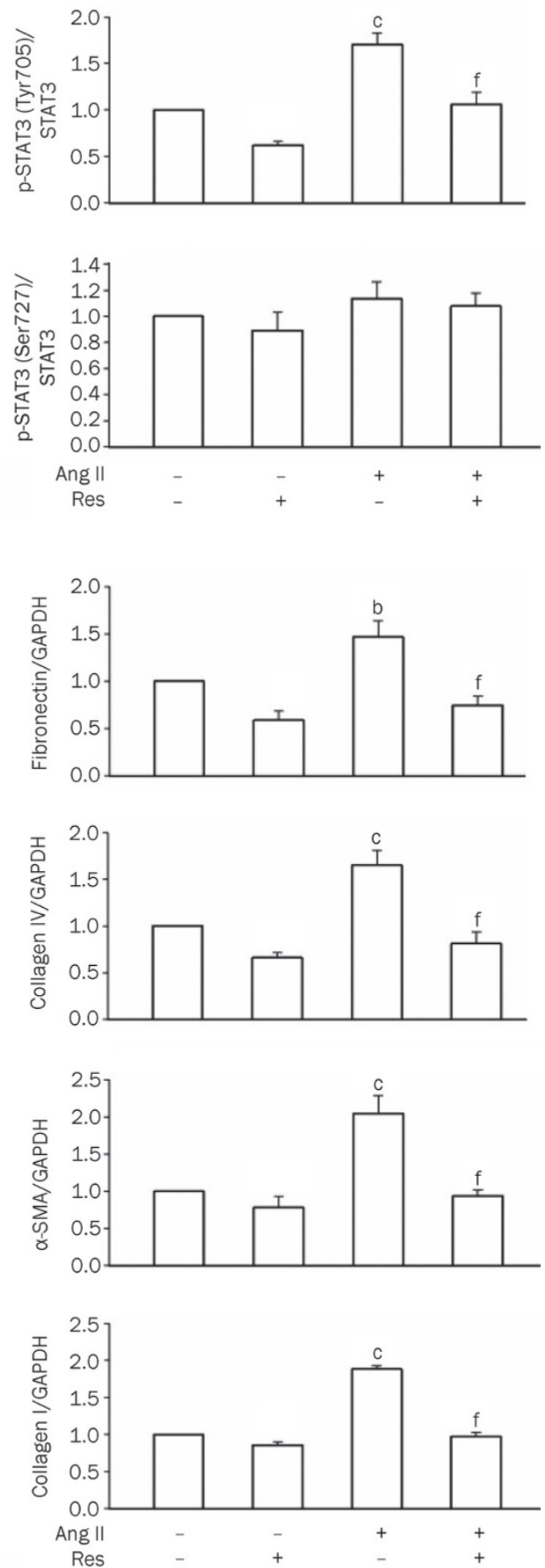

Figure 3. Effect of resveratrol (Res) on Ang II-induced STAT3 activation and expression of pro-fibrotic genes in NRK-52E cells. Cells were treated with Ang II in the presence or absence of Res. (A) After $1 \mathrm{~h}$ of Ang II treatment, with or without $1 \mathrm{~h}$ Res (12.5 $\mu$ mol/L) pretreatment, acetyl-STAT3 (Lys685), phospho-STAT3 (Tyr705) and phospho-STAT3 (Ser727) were analyzed by Western blot analysis. (B) After $48 \mathrm{~h}$ of Ang II treatment, with or without $1 \mathrm{~h}$ Res $(12.5 \mu \mathrm{mol} / \mathrm{L})$ pretreatment, fibronectin, collagen I, collagen IV, and $\alpha$-SMA were analyzed by Western blot analysis. Media containing Ang II or Res were changed every $24 \mathrm{~h}$. Data are the mean \pm SEM of 4 experiments. ${ }^{b} P<0.05,{ }^{\mathrm{C}} P<0.01$ compared with control [Ang II $(-)$ and Res $(-)$ ]. ${ }^{\text {f }} P<0.01$ compared with Ang II only. 
A

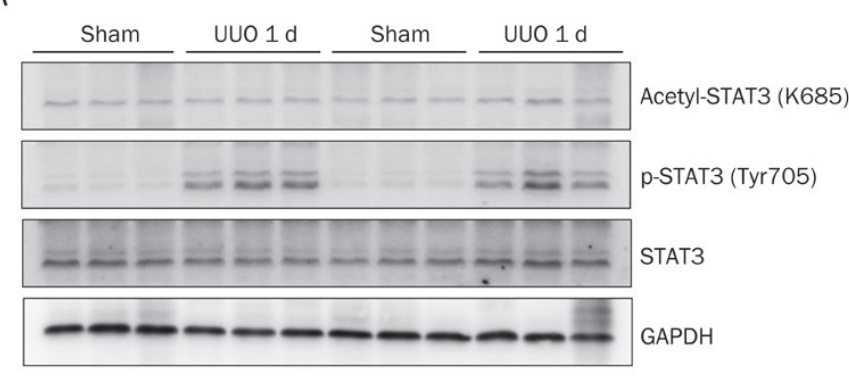

B

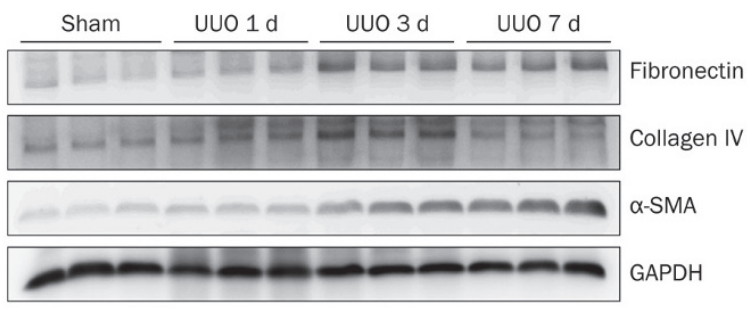

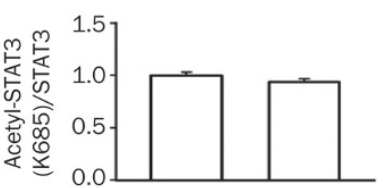
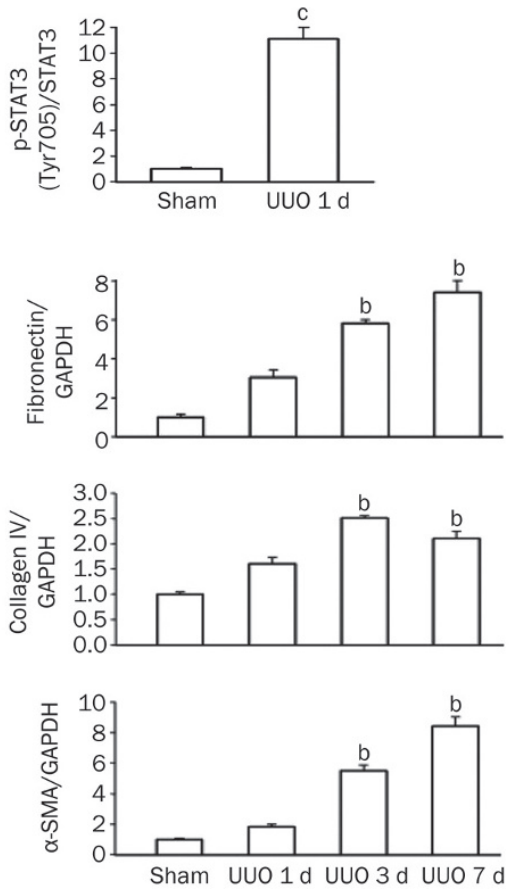

Figure 4. Unilateral ureteral obstruction (UUO) induced pro-fibrotic responses in the kidney. (A) Acetyl-STAT3 (Lys685) and phospho-STAT3 (Tyr705) were analyzed by Western blot analysis after UUO for $1 \mathrm{~d}$. (B) Western blotting visualized fibronectin, collagen IV and $\alpha$-SMA in a sample time course of UUO mice. Data are the mean \pm SEM of 6 animals. ${ }^{b} P<0.05$ compared with sham.

brotic effect that was similar to in vitro observations. To our knowledge, this is the first evidence suggesting that STAT3 acetylation is involved in renal fibrosis in vivo. Both in vitro and in vivo findings indicate that STAT3 acetylation facilitates phosphorylation at special sites, such as Try705. This conclusion is consistent with those of previous studies. For instance, it has been proven that SIRT1 opposes STAT3 phosphorylation and STAT3-dependent effects of IL-22 through STAT3 deacetylation in keratinocytes ${ }^{[39]}$. In addition, trichostatin $\mathrm{A}, \mathrm{a}$ broad inhibitor of class I and class II HDACs ${ }^{[51,52]}$, can abolish the inhibition of STAT3 activation that is induced by class I HDAC overexpression in $293 \mathrm{~T}$ cells, indicating that acetylation is critical for STAT3 activation ${ }^{[32]}$. The potential mechanism of the interaction between STAT3 acetylation and phosphorylation may be the sustained stabilization of STAT3 provided by acetylation resulting from the blockage of degradation via the ubiquitination-mediated proteasomal pathway ${ }^{[53]}$.

The exact role that HDACs or acetylated STAT3 plays in renal fibrosis seems to be much more complicated than the features presented by the current study. Therefore, it is difficult to claim that the antifibrotic effect of resveratrol on the development of renal fibrosis is entirely due to the inhibition of STAT3 activation. Further studies using more selective approaches need to be performed to determine whether antifibrotic actions are simply attributed to reducing STAT3 acetylation and the interaction between STAT3 acetylation and phosphorylation under physiological and pathophysiological circumstances. However, the similar molecular mechanisms of resveratrol's antifibrotic effects identified in vitro and in vivo suggest the essential role of STAT3 acetylation in renal profibrotic responses.

In this study, we demonstrated a constitutive STAT3 acetylation on Lys685 in the kidney that may be indispensable for STAT3 signaling-mediated fibrogenesis. These findings suggest that the inhibition of STAT3 acetylation may represent a novel therapeutic approach toward CKD associated with fibrosis.

\section{Acknowledgements}

This research was financially supported by the National Natural Science Foundation of China (No 81070577, 81170636) to Li-min LU, (No 81000280$)$ to Hong XUE, (No 81100531$)$ to Wei ZHANG and (№ 81070547) to Chen YU.

\section{Author contribution}

Yu HUANG, Wei ZHANG, Chen YU, and Li-min LU designed the research project; Jun NI and Yang SHEN performed the experiments; Zhen WANG, De-cui SHAO, and Jia LIU ana- 

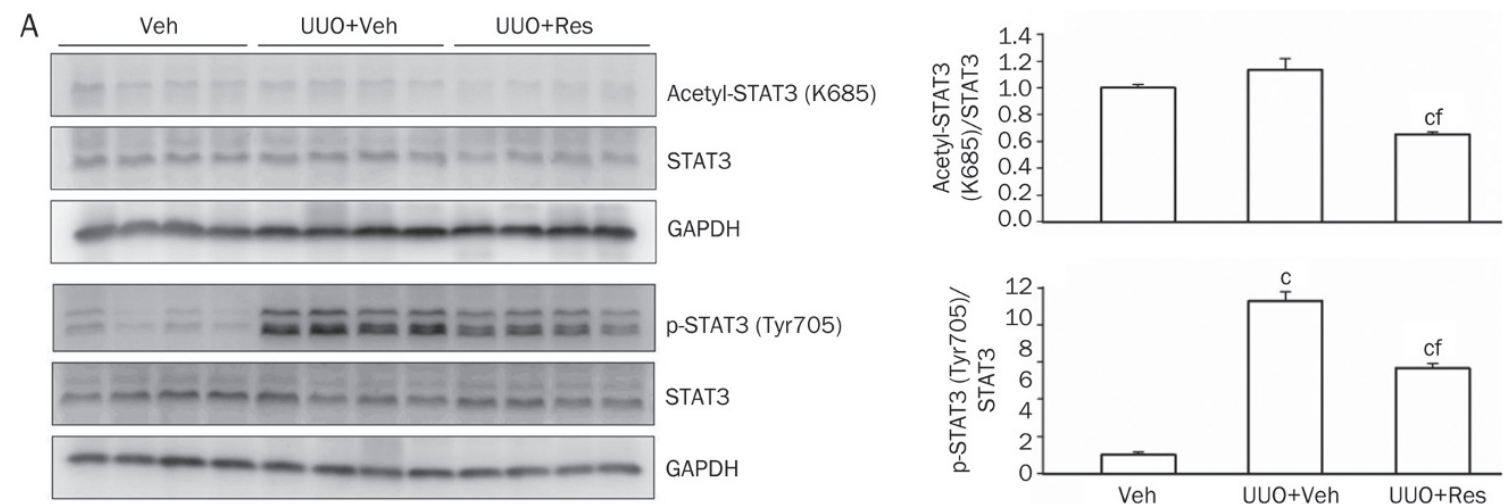

B
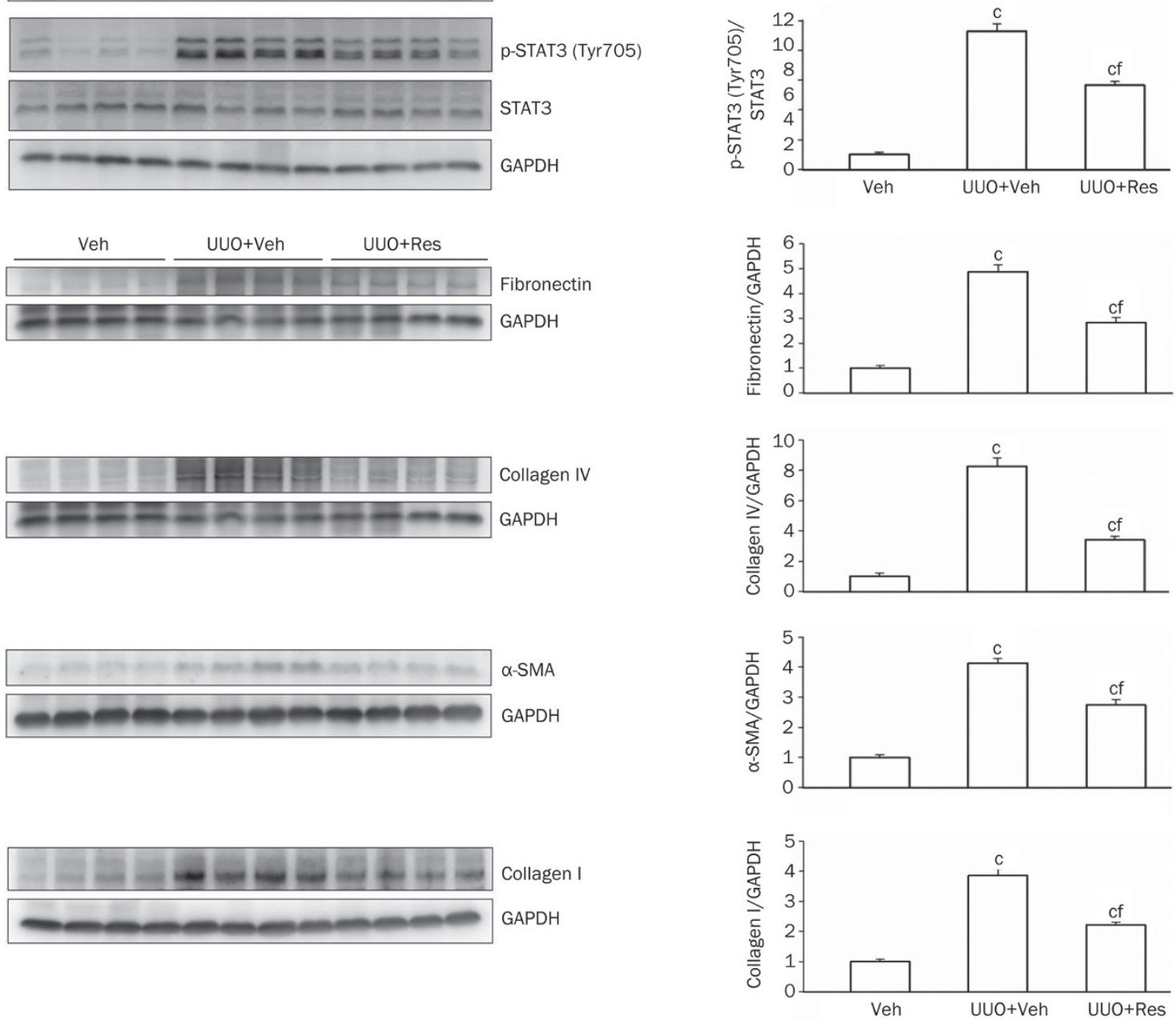

Figure 5. Effect of Res on UUO-induced STAT3 activation and expression of pro-fibrotic genes in the obstructed kidney. Control and UUO mice received vehicle or $100 \mathrm{mg} / \mathrm{kg}$ Res by oral gavage once a day for $1 \mathrm{~d}$ (A) or $3 \mathrm{~d}$ (B). (A) Acetyl-STAT3 (Lys685) and phospho-STAT3 (Tyr705) were analyzed by Western blot analysis. (B) Fibronectin, collagen IV, $\alpha$-SMA, and collagen I were analyzed by Western blot analysis. Data are the mean \pm SEM of 4 animals. ${ }^{\mathrm{C}} P<0.01$ compared with vehicle (Veh). ${ }^{\mathrm{f}} P<0.01$ compared with UUO with vehicle (UUO+Veh).

lyzed the data; Lan-jun FU and Ya-li KONG contributed the reagents and materials; Jun NI, Li ZHOU, and Hong XUE wrote the manuscript.

\section{References}

1 Eddy AA. The STAT3 $\mathrm{NH}_{2}$-terminal domain stabilizes enhanceosome assembly by interacting with the p300 bromodomain. Adv Chronic Kidney Dis 2005; 12: 353-65.

2 Liu Y. Renal fibrosis: new insights into the pathogenesis and therapeutics. Kidney Int 2006; 69: 213-7.

3 Matsui F, Rhee A, Hile KL, Zhang H, Meldrum KK. IL-18 induces profibrotic renal tubular cell injury via STAT3 activation. 2013: 305; F1014-F1021.

4 Iwano M, Plieth D, Danoff TM, Xue C, Okada H, Neilson EG. Evidence that fibroblasts derive from epithelium during tissue fibrosis. J Clin Invest 2002; 110: 341-50.

5 Liu Y. Epithelial to mesenchymal transition in renal fibrogenesis: pathologic significance, molecular mechanism, and therapeutic intervention. J Am Soc Nephrol 2004; 15: 1-12.

6 Rastaldi MP, Ferrario F, Giardino L, Dell'Antonio G, Grillo C, Grillo P, et al. Epithelial-mesenchymal transition of tubular epithelial cells in human renal biopsies. Kidney Int 2002; 62: 137-46.

7 Zeisberg M, Hanai J, Sugimoto H, Mammoto T, Charytan D, Strutz F, et al. BMP-7 counteracts TGF-beta1-induced epithelial-to-mesenchymal transition and reverses chronic renal injury. Nat Med 2003; 9: 9648.

8 Ivanova L, Butt MJ, Matsell DG. Mesenchymal transition in kidney collecting duct epithelial cells. Am J Physiol Renal Physiol 2008; 294: 
A

정

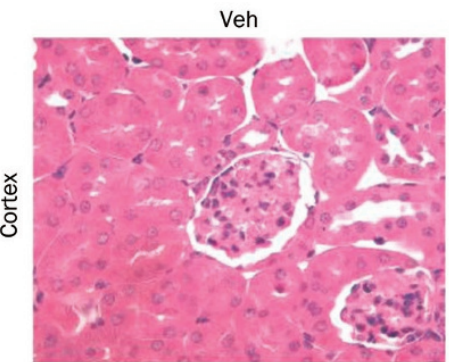

또

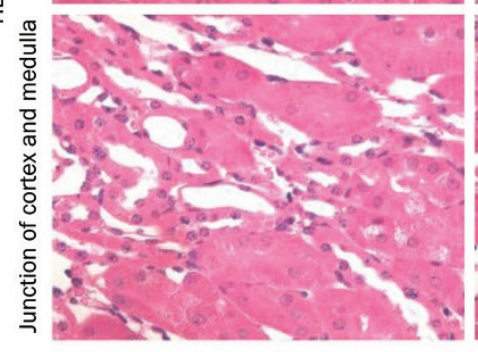

B
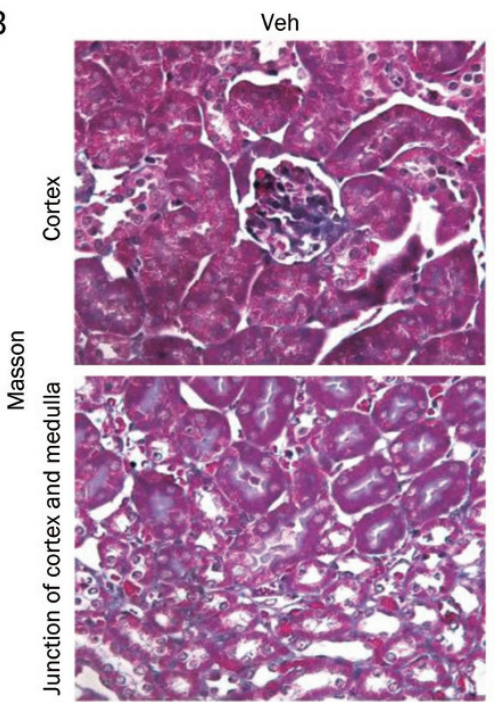
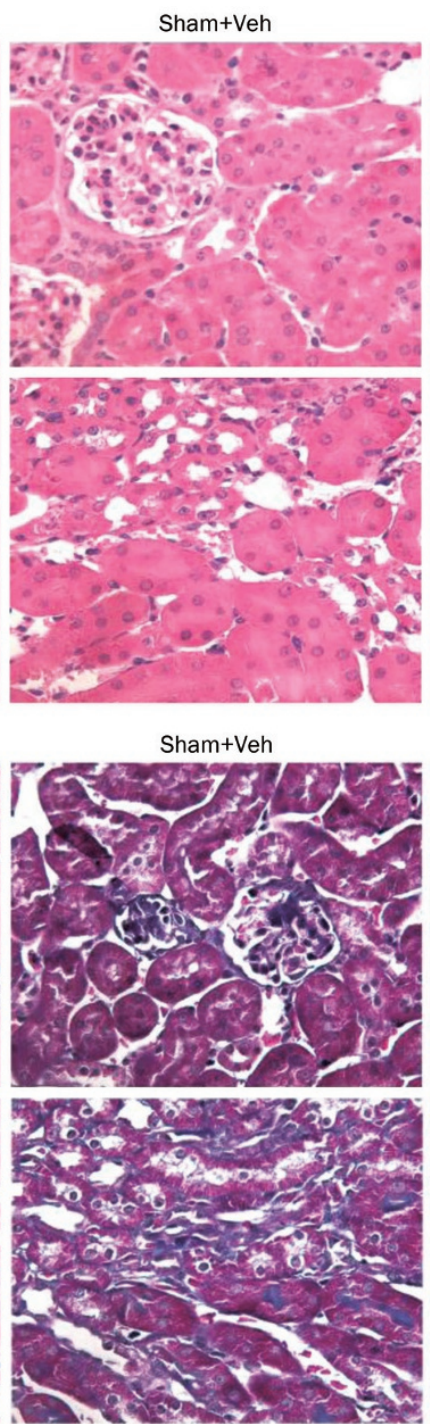

UUO+Veh
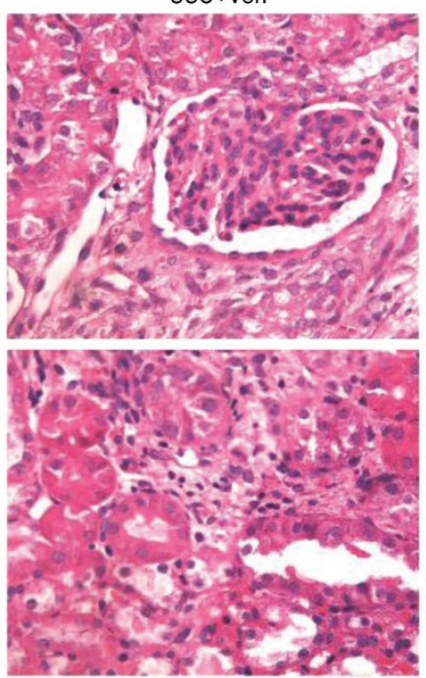
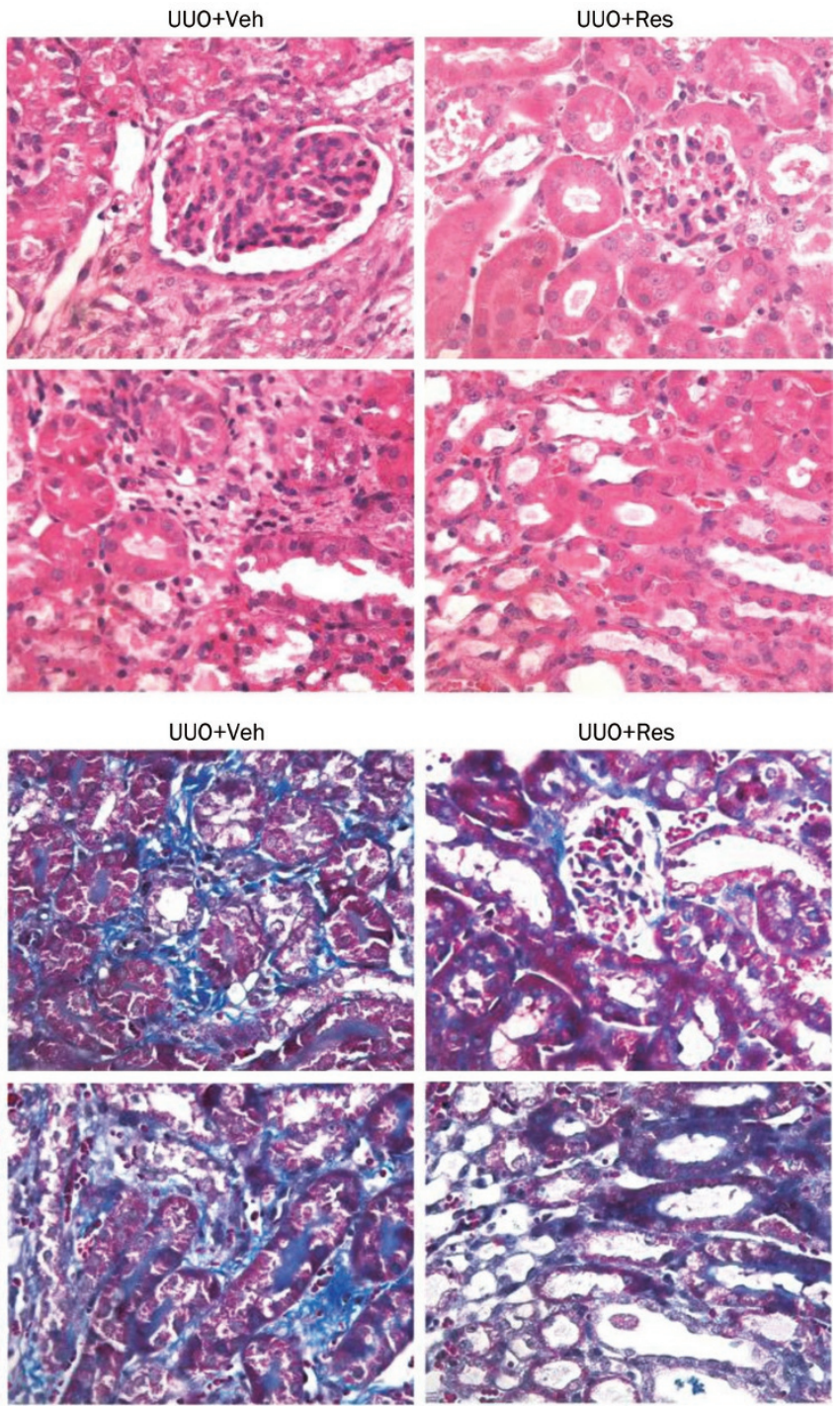

Figure 6. Effect of Res on tubulointerstitial fibrosis in the UUO kidney. Control, sham and UUO mice received vehicle or $100 \mathrm{mg} / \mathrm{kg}$ Res by oral gavage once a day for $3 \mathrm{~d}$. (A) Representative sections of hematoxylin and eosin (HE)-stained kidneys. Original magnifications $\times 400$. (B) Representative sections of Masson's trichrome-stained kidneys. Collagen was stained blue. Original magnifications $\times 400$.

\section{F1238-F1248.}

9 Ranganathan P, Jayakumar C, Ramesh G. Proximal tubule-specific overexpression of netrin-1 suppresses acute kidney injury-induced interstitial fibrosis and glomerulosclerosis through suppression of IL-6/STAT3 signaling. Am J Physiol Renal Physiol 2013; 304: F1054F1065.

10 Uchida Y, Miyajima A, Kikuchi E, Kozakai N, Kosaka T, leda M, et al. Renal damage inhibited in mice lacking angiotensinogen gene subjected to unilateral ureteral obstruction. Urology 2009; 74: 93843.

11 Grande MT, Perez-Barriocanal F, Lopez-Novoa J M. Role of inflammation in tubulo-interstitial damage associated to obstructive nephropathy. J Inflamm (Lond) 2010; 7: 19.

12 Tang SC, Lai KN. The pathogenic role of the renal proximal tubular cell in diabetic nephropathy. Nephrol Dial Transplant 2012; 27: 304956.

13 Zhong J, Guo D, Chen CB, Wang W, Schuster M, Loibner H, et al. Prevention of angiotensin II-mediated renal oxidative stress, inflammation, and fibrosis by angiotensin-converting enzyme 2 . Hypertension 2011; 57: 314-22.

14 Alberti C. Congenital ureteropelvic junction obstruction: physiopathology, decoupling of tout court pelvic dilatation-obstruction semantic connection, biomarkers to predict renal damage evolution. Eur Rev Med Pharmacol Sci 2012; 16: 213-9.

15 El CM, Chen J, Seshan SV, Jha S, Richardson I, Ledbetter SR, et al. Effect of combination therapy with enalapril and the TGF-beta antagonist 1D11 in unilateral ureteral obstruction. Am J Physiol Renal Physiol 2007; 292: F1291-F1301.

16 Shin GT, Kim WH, Yim H, Kim MS, Kim H. Effects of suppressing intrarenal angiotensinogen on renal transforming growth factor-beta1 expression in acute ureteral obstruction. Kidney Int 2005; 67: $897-$ 908.

17 Terashima H, Kato M, Yasumo H, Tsuchida H, Mizuno M, Sada T. A sensitive short-term evaluation of antifibrotic effects using newly established type I collagen reporter transgenic rats. Am J Physiol Renal Physiol 2010; 299: F792-F801. 
18 Kodama H, Fukuda K, Pan J, Makino S, Sano M, Takahashi T, et al. Biphasic activation of the JAK/STAT pathway by angiotensin II in rat cardiomyocytes. Circ Res 1998; 82: 244-50.

19 Weng YI, Aroor AR, Shukla SD. Ethanol inhibition of angiotensin IIstimulated Tyr705 and Ser727 STAT3 phosphorylation in cultured rat hepatocytes: relevance to activation of $\mathrm{p} 42 / 44$ mitogen-activated protein kinase. Alcohol 2008; 42: 397-406.

20 Kandalam U, Clark MA. Angiotensin II activates JAK2/STAT3 pathway and induces interleukin- 6 production in cultured rat brainstem astrocytes. Regul Pept 2010; 159: 110-6.

21 Amiri F, Shaw S, Wang X, Tang J, Waller JL, Eaton DC, et al. Angiotensin II activation of the JAK/STAT pathway in mesangial cells is altered by high glucose. Kidney Int 2002; 61: 1605-16.

22 Chen X, Wang J, Zhou F, Wang X, Feng Z. STAT proteins mediate angiotensin II-induced production of TIMP-1 in human proximal tubular epithelial cells. Kidney Int 2003; 64: 459-67.

23 Bromberg J, Darnell JJ. The role of STATs in transcriptional control and their impact on cellular function. Oncogene 2000; 19: 2468-73.

24 Liu DB, Hu GY, Long GX, Qiu H, Mei Q, Hu GQ. Celecoxib induces apoptosis and cell-cycle arrest in nasopharyngeal carcinoma cell lines via inhibition of STAT3 phosphorylation. Acta Pharmacol Sin 2012; 33: 682-90.

25 Kuratsune M, Masaki T, Hirai T, Kiribayashi K, Yokoyama Y, Arakawa T, et al. Signal transducer and activator of transcription 3 involvement in the development of renal interstitial fibrosis after unilateral ureteral obstruction. Nephrology (Carlton) 2007; 12: 565-71.

26 Pang M, Kothapally J, Mao H, Tolbert E, Ponnusamy M, Chin YE, et al. Inhibition of histone deacetylase activity attenuates renal fibroblast activation and interstitial fibrosis in obstructive nephropathy. Am J Physiol Renal Physiol 2009; 297: F996-F1005.

27 Liu N, Guo JK, Pang M, Tolbert E, Ponnusamy M, Gong R, et al. Genetic or pharmacologic blockade of EGFR inhibits renal fibrosis. J Am Soc Nephrol 2012; 23: 854-67.

28 Giannopoulou M, Iszkula SC, Dai C, Tan X, Yang J, Michalopoulos GK, et al. Distinctive role of Stat3 and Erk-1/2 activation in mediating interferon-gamma inhibition of TGF-beta1 action. Am J Physiol Renal Physiol 2006; 290: F1234-F1240.

29 Sarkozi R, Flucher K, Haller VM, Pirklbauer M, Mayer G, Schramek H. Oncostatin M inhibits TGF-beta1-induced CTGF expression via STAT3 in human proximal tubular cells. Biochem Biophys Res Commun 2012; 424: 801-6.

30 Wen Z, Zhong Z, Darnell JJ. Maximal activation of transcription by Stat1 and Stat3 requires both tyrosine and serine phosphorylation. Cell 1995; 82: 241-50.

31 Wang R, Cherukuri P, Luo J. Activation of Stat3 sequence-specific DNA binding and transcription by $\mathrm{p} 300 / \mathrm{CREB}-$ binding protein-mediated acetylation. J Biol Chem 2005; 280: 11528-34.

32 Yuan Z L, Guan YJ, Chatterjee D, Chin YE. Stat3 dimerization regulated by reversible acetylation of a single lysine residue. Science 2005; 307: 269-73.

33 Ray S, Sherman CT, Lu M, Brasier AR. Angiotensinogen gene expression is dependent on signal transducer and activator of transcription 3-mediated p300/cAMP response element binding protein-binding protein coactivator recruitment and histone acetyltransferase activity. Mol Endocrinol 2002; 16: 824-36.

34 Pang M, Ma L, Liu N, Ponnusamy M, Zhao TC, Yan H, et al. Histone deacetylase $1 / 2$ mediates proliferation of renal interstitial fibroblasts and expression of cell cycle proteins. J Cell Biochem 2011; 112: 2138-48.

35 Denu JM. The Sir 2 family of protein deacetylases. Curr Opin Chem Biol 2005; 9: 431-40.
36 Das C, Lucia MS, Hansen KC, Tyler JK. CBP/p300-mediated acetylation of histone $\mathrm{H} 3$ on lysine 56. Nature 2009; 459: 113-7.

37 Nie Y, Erion DM, Yuan Z, Dietrich M, Shulman GI, Horvath TL, et al. STAT3 inhibition of gluconeogenesis is downregulated by SirT1. Nat Cell Biol 2009; 11: 492-500.

38 Erion DM, Yonemitsu S, Nie Y, Nagai Y, Gillum MP, Hsiao JJ, et al. SirT1 knockdown in liver decreases basal hepatic glucose production and increases hepatic insulin responsiveness in diabetic rats. Proc Natl Acad Sci U S A 2009; 106: 11288-93.

39 Sestito R, Madonna S, Scarponi C, Cianfarani F, Failla CM, Cavani A, et al. STAT3-dependent effects of IL-22 in human keratinocytes are counterregulated by sirtuin 1 through a direct inhibition of STAT3 acetylation. FASEB J 2011; 25: 916-27.

40 Schenk S, Mccurdy CE, Philp A, Chen MZ, Holliday MJ, Bandyopadhyay GK, et al. Sirt1 enhances skeletal muscle insulin sensitivity in mice during caloric restriction. J Clin Invest 2011; 121: 4281-8.

41 Scuto A, Kirschbaum M, Buettner R, Kujawski M, Cermak JM, Atadja $P$, et al. SIRT1 activation enhances HDAC inhibition-mediated upregulation of GADD45G by repressing the binding of NF-kappaB/ STAT3 complex to its promoter in malignant lymphoid cells. Cell Death Dis 2013; 4: e635.

42 Howitz KT, Bitterman KJ, Cohen HY, Lamming DW, Lavu S, Wood JG, et al. Small molecule activators of sirtuins extend Saccharomyces cerevisiae lifespan. Nature 2003; 425: 191-6.

43 Fu Y, Wang Y, Du L, Xu C, Cao J, Fan T, et al. Resveratrol inhibits ionising irradiation-induced inflammation in MSCs by activating SIRT1 and limiting NLRP-3 inflammasome activation. Int J Mol Sci 2013; 14: 14105-18.

44 Li J, Qu X, Ricardo SD, Bertram JF, Nikolic-Paterson DJ. Resveratrol inhibits renal fibrosis in the obstructed kidney: potential role in deacetylation of Smad3. Am J Pathol 2010; 177: 1065-71.

45 Kim DH, Jung YJ, Lee JE, Lee AS, Kang KP, Lee S, et al. SIRT1 activation by resveratrol ameliorates cisplatin-induced renal injury through deacetylation of p53. Am J Physiol Renal Physiol 2011; 301: F427F435.

46 Jung YJ, Lee JE, Lee AS, Kang KP, Lee S, Park SK, et al. SIRT1 overexpression decreases cisplatin-induced acetylation of NF-kappaB p65 subunit and cytotoxicity in renal proximal tubule cells. Biochem Biophys Res Commun 2012; 419: 206-10.

47 He M, Zhang L, Shao Y, Xue H, Zhou L, Wang XF, et al. Angiotensin II type 2 receptor mediated angiotensin II and high glucose induced decrease in renal prorenin/renin receptor expression. Mol Cell Endocrinol 2010; 315: 188-94.

48 Imai S, Armstrong CM, Kaeberlein M, Guarente L. Transcriptional silencing and longevity protein Sir2 is an NAD-dependent histone deacetylase. Nature 2000; 403: 795-800.

49 Landry J, Sutton A, Tafrov ST, Heller RC, Stebbins J, Pillus L, et al. The silencing protein SIR2 and its homologs are NAD-dependent protein deacetylases. Proc Natl Acad Sci U S A 2000; 97: 5807-11.

50 North BJ, Marshall BL, Borra MT, Denu JM, Verdin E. The human Sir2 ortholog, SIRT2, is an NAD+-dependent tubulin deacetylase. Mol Cell 2003; 11: 437-44.

51 Yoshida M, Horinouchi S, Beppu T. Trichostatin A and trapoxin: novel chemical probes for the role of histone acetylation in chromatin structure and function. Bioessays 1995; 17: 423-30.

52 Monneret C. Histone deacetylase inhibitors. Eur J Med Chem 2005; 40: $1-13$.

53 Jain S, Wei J, Mitrani LR, Bishopric NH. Auto-acetylation stabilizes p300 in cardiac myocytes during acute oxidative stress, promoting STAT3 accumulation and cell survival. Breast Cancer Res Treat 2012; 135: $103-14$. 